million (ppm) using a QuinTron BreathTracker DP at baseline, then-after $75 \mathrm{~g}$ oral glucose-at $20 \mathrm{~min}$ time points up to $3 \mathrm{~h}$ or until positive. Positive test: fasting $\mathrm{H}_{2} \geq 20$ or $\mathrm{CH}_{4} \geq 10 \mathrm{ppm}$ or a rise in $\mathrm{H}_{2} \geq 12$ or $\mathrm{CH}_{4} \geq 6 \mathrm{ppm}$. Some patients also had duodenal aspirates collected endoscopically. Positive result: $>10^{4}$ colony forming units $/ \mathrm{ml}$. Patients with positive tests were treated with antibiotics. Results were assessed retrospectively.

Results 126 patients, 66 males and 60 females, median group age 61 years (range 35-86), treated for gynaecological (30\%), upper GI (25\%), lower GI $(9 \%)$, urological $(28 \%)$, other cancers $(10 \%-$ myeloma, ependymoma, bronchial, breast and lymphoma) were referred. Daily troublesome GI symptoms included flatulence (85\%), borborygmi (63\%), belching (54\%), bloating (52\%), abdominal pain $(50 \%)$, steatorrhoea $(38 \%)$, nausea/vomiting $(23 \%)$ and diarrhoea $(19 \%) .60(48 \%)$ had a positive breath test $-5 \% \mathrm{H} 2,10 \% \mathrm{CH} 4$ and $33 \%$ both gases. $21(17 \%)$ of 86 patients with D2 aspirates tested positive for Streptococcus ( $\mathrm{n}=6)$, E. Coli (3), Candida (3), Klebsiella (2), Enterococcus (2), Pseudomonas (2), Neisseria (1), Aeromonas (1) and Stenotrophomonas (1). 17 (33\%) had negative breath tests. Six of these had positive D2 aspirates with $3(50 \%)$ responsive to antibiotics, 11 had negative aspirates but a $73 \%$ antibiotic response rate. $24(46 \%)$ tested positive for both gases. 33\% of these had a positive D2 aspirate with a $75 \%$ response rate to antibiotics and $67 \%$ negative aspirates but $81 \%$ response. Six patients (12\%) were positive only for $\mathrm{H} 2$ (all had negative aspirates), 1 responded to antibiotics. $5(10 \%)$ tested positive for $\mathrm{CH} 4$ only, of which 1 had a positive D2 aspirate with a response to antibiotics and $4(80 \%)$ had negative aspirate but 2 responded.

Conclusion Methane breath testing identifies 10\% more patients with SIBO compared to the glucose hydrogen breath test alone. D2 aspirate increases the detection rate by $12 \%$. A trial of antibiotics, with other tests negative, benefits $15 \%$ of patients. All current diagnostic methods are flawed. Better diagnostic tests for SIBO are required.

Competing interests None declared.

\section{PTU-156 DIETARY RESPONSE IN COELIAC DISEASE SHOULD BE ASSESSED BY REPEAT DUODENAL BIOPSY}

\section{doi:10.1136/gutjnl-2012-302514c.156}

${ }^{1} \mathrm{G}$ Corbett, ${ }^{1} \mathrm{~L}$ Sharkey, ${ }^{2} \mathrm{E}$ Currie, ${ }^{2} \mathrm{~J}$ Lee, ${ }^{3} \mathrm{~N}$ Sweeney, ${ }^{1} \mathrm{~J}$ Woodward. ${ }^{1}$ Department of Gastroenterology and Clinical Nutrition, Addenbrookes Hospital, Cambridge, UK; ${ }^{2}$ Department of Nutrition and Dietetics, Addenbrookes Hospital, Cambridge, UK; ${ }^{3}$ Univeristy of Adelaide, Adelaide, Australia

Introduction Therapeutic goals in coeliac disease (CD) are poorly defined. Emerging evidence reports worse outcomes with lack of mucosal recovery, suggesting histological normalisation as the aim of treatment. However, few centres routinely re-biopsy and many clinicians depend on serology or symptoms for follow-up. This retrospective study evaluates the role of the follow-up biopsy compared to serology in treated coeliacs and is the largest study of paired biopsies reported.

Methods Details of patients attending the Addenbrookes Adult CD Clinic are entered onto a database. Patients undergo repeat duodenal biopsy 9-12 months after commencing dietary therapy. Serum Anti-TTG antibodies and total IgA are measured at clinic visits. From the database of around 600 adult patients (30\% male, $70 \%$ female, average age at diagnosis 48 and 44 yrs respectively) followup biopsy results were available for 433 patients and compared using a paired t-test.

Results Paired initial and follow-up biopsies were available for 368/ 433 patients (no information about index biopsy in 65). 239 (65\%) were re-biopsied within 12 months, 311 (85\%) within 24 months. The proportion achieving normalisation of duodenal mucosa (Marsh 0) at
12 months, $12-24$ months or after 24 months is $27 \%, 25 \%(p=0.61)$ and $25 \%(\mathrm{p}=0.1)$ respectively. Those without villous atrophy (VA) (Marsh 0,1 or 2$)$ is $58 \%, 58 \%(p=0.98)$ and $52 \%(p=0.24)$ respectively. Excluding the $2.5 \%$ of patients with $\operatorname{IgA}$ deficiency, $13 \%$ of patients had negative anti-TTG antibody titres at the time of diagnostic biopsy. In the group of patients with positive serology at first biopsy, only $43 \%$ with VA on follow-up biopsy were positive for anti-TTG antibodies. Conversely, 13\% without VA on follow-up had positive serology. The sensitivity, positive predictive and negative predictive values for serology compared to biopsy at follow-up are $43.4 \%, 68.8 \%$ and $70.5 \%$ respectively.

Conclusion Response to diet in CD might be monitored by symptoms, serology or histology. Many patients diagnosed in adulthood report minimal or no symptoms at diagnosis and this is therefore not a credible marker of response. Here we show that:

- Anti-TTG antibody serology has a sensitivity of $87 \%$ at diagnosis

- Anti-TTG serology has a poor sensitivity (43\%) on follow-up and is therefore unreliable

- Histological response rates are not increased after 1 year on diet

- Approximately $28 \%$ of patients achieve normal duodenal mucosa, $58 \%$ show no or minimal signs of coeliac disease on follow-up biopsy.

We suggest that follow-up biopsy on diet is the only reliable form of assessing response to treatment, and that the proportion of patients achieving histological response could be used as a means of auditing quality of care in coeliac clinics.

Competing interests None declared.

\section{PTU-157 A PROSPECTIVE STUDY OF SERUM VITAMIN A AND D LEVELS IN PRIMARY BILE ACID DIARRHOEA}

doi:10.1136/gutjnl-2012-302514c.157

II Johnston, * 'S S Pattni, ${ }^{1} \mathrm{~J} D$ Nolan, ${ }^{2} \mathrm{~T}$ Dew, ${ }^{1} \mathrm{~J} R$ Walters. ${ }^{1}$ Gastroenterology and Hepatology, Imperial College London, London, UK; ${ }^{2}$ Clinical Chemistry, Kings College London NHS Trust, London, UK

Introduction There is evidence that primary bile acid diarrhoea (BAD) is caused by disruption of the homeostatic mechanism controlling bile acid synthesis, with an overproduction of bile acids leading to chronic watery diarrhoea. Fibroblast Growth Factor 19 (FGF19) has been shown to be central to this homeostatic mechanism, providing negative feedback on CYP7A1, the rate limiting enzyme in bile acid synthesis. Median serum FGF19 levels have been shown to be lower in $\mathrm{BAD}$ patients than in diarrhoea controls. In mouse models, it has also been shown that expression of FGF15, the orthologue of FGF19, is induced by the active forms of vitamins A and $\mathrm{D}$. The aim of this study was to investigate a possible causal relationship between low serum levels of vitamins $A$ and $D$ and $\mathrm{BAD}$.

Methods Patients with chronic diarrhoea were recruited prospectively. All patients underwent routine testing to exclude other causes of diarrhoea and had SeHCAT tests. Patients were classified as having primary $\mathrm{BAD}$, secondary $\mathrm{BAD}$, or unexplained chronic diarrhoea (CD). Serum $25 \mathrm{OH}$-vitamin D was measured by LC-MS using standard procedures. Serum Vitamin A was calculated by HPLC. Results are expressed as medians. Mann-Whitney and Spearman rank correlation tests were used in analyses.

Results Serum vitamin A levels were available in 145 subjects (primary BAD 46; secondary 29; CD 70). Serum vitamin D levels were available in 150 subjects (primary BAD 50; secondary 29; CD 71). There were no significant differences in the level of vitamin $A$ between the groups with primary or secondary BAD or CD (2.10, 
1.90 and $1.95 \mathrm{umol} / 1$ respectively, $\mathrm{p}>0.05)$. There was no significant correlation of vitamin A with FGF19 or SeHCAT retention as a whole or within the subgroups. There were no significant differences in serum 25OH-vitamin $\mathrm{D}$ between the groups (38, 47, $46 \mathrm{nmol} / 1, \mathrm{p}>0.05)$ There was no correlation of $25 \mathrm{OH}$-vitamin $\mathrm{D}$ with FGF19 or SeHCAT retention as a whole, or within the subgroups.

Conclusion As vitamins $\mathrm{A}$ and $\mathrm{D}$ are known to induce FGF15 expression in mice, it was important to establish whether these vitamins could be associated with the abnormal FGF19 levels seen in human $\mathrm{BAD}$. These results do not provide support to the possibility that deficiency of these vitamins is directly involved in the pathogenesis of $\mathrm{BAD}$.

Competing interests None declared.

\section{PTU-158 COELIAC DISEASE INVESTIGATION AND FOLLOW-UP}

doi:10.1136/gutjnl-2012-302514c.158

${ }^{1} \mathrm{I}$ Nasr, ${ }^{*}{ }^{1} \mathrm{M}$ Austin, ${ }^{2} \mathrm{M}$ Tarzi. ${ }^{1}$ Gastroenterology, Brighton and Sussex University Hospital, Brighton, UK; ${ }^{2}$ Immunology, Brighton and Sussex University Hospital, Brighton, UK

Introduction Coeliac disease (CD) is an abnormal immune response to gluten affecting $1 \%$ of the UK population, resulting in small bowel villous atrophy, malabsorption and GI symptoms. A large number of patients are tested for $\mathrm{CD}$ and often repeat requests are made. This retrospective analysis looked at the prevalence of patients with positive CD serology in relation to subsequent gastroenterology referrals and/or small bowel biopsy.

Methods The data were collected from patients who had undergone serological CD testing. All patients with a positive anti-tissue transglutaminase, or a positive endomysial antibody test were recorded and notes analysed for referral or endoscopy. We excluded patients under 18 years of age and those with known CD. The data were obtained from the laboratory database at Brighton and Sussex University Hospitals over a period of 1 year.

Results We identified 7369 CD serology requests. The referral source for CD serology was a general practitioner, hospital specialist, gastrointestinal surgeon or gastroenterologist. Overall, 169 (2.3\%) individuals had a positive result. From the total number of requests, $6.75 \%$ (498) were repeat requests. Of these, $8.23 \%$ (41) had a second positive test. $13.86 \%$ (69) of the patients who had a repeat serology done by a general practitioner or other hospital clinician were referred to gastroenterology. The overall prevalence of CD was $2.27 \%$ (167), of which 58\% (97) were referred for a small bowel biopsy. Small bowel histology confirmed CD in $52 \%$ of the biopsy samples, that is, almost one third of the total number of cases with positive serology.

Conclusion Positive CD serology was recorded in $2.3 \%$ of the study group. Although small bowel histology is the gold standard in the diagnosis of $\mathrm{CD},{ }^{1}$ this was performed on $58 \%$ of the patients with positive serology. It is apparent that not all cases with positive serology are being referred to a specialist. We recommend that

Abstract PTU-158 Table 1 Source of coeliac serology referral

\begin{tabular}{lcrr}
\hline & $\begin{array}{l}\text { Total serology } \\
\text { requests (\%) }\end{array}$ & $\begin{array}{l}\text { Positive serology } \\
\text { result (\%) }\end{array}$ & \multicolumn{1}{l}{$\begin{array}{l}\text { Negative serology } \\
\text { result (\%) }\end{array}$} \\
\hline GP & $4502(61.1 \%)$ & $82 / 4502(1.8 \%)$ & $4420 / 4502(98.2 \%)$ \\
Other hospital specialist & $1716(23.3 \%)$ & $41 / 1716(2.4 \%)$ & $1675 / 1716(97.6 \%)$ \\
Gl surgeon & $102(1.4 \%)$ & $3 / 102(2.9 \%)$ & $99 / 102(97.1 \%)$ \\
Gastroenterology & $1049(14.2 \%)$ & $41 / 1049(3.9 \%)$ & $10081049(96.1 \%)$ \\
\hline
\end{tabular}

patients with suspected or incidental diagnosis of CD should be referred to a gastroenterologist for assessment, confirmation of the diagnosis with small bowel biopsy and advice on gluten-free diet.

Competing interests None declared.

\section{REFERENCES}

1. Ciclitira PJ, Dewar DH, McLaughlin SD, et al. The management of adults with coeliac disease.

2. Crowe SE. Celiac Disease: When to Look and How? ACG 2011 Annual PG Course.

\section{PTU-159 SELF REPORTING OF GLUTEN SENSITIVE GI SYMPTOMS IN PRIMARY CARE: SHOULD WE ACCEPT THE DIAGNOSIS OF COELIAC DISEASE WITHOUT FURTHER INVESTIGATIONS?}

doi:10.1136/gutjnl-2012-302514c.159

I Aziz, ${ }^{*}$ K E Evans, L Newrick, D S Sanders. Department of Gastroenterology, Royal Hallamshire Hospital, Sheffield, UK

Introduction Many unselected patients presenting to gastrointestinal (GI) clinics self-report that they have symptoms related to the ingestion of gluten. For this reason we undertook a prospective and systematic evaluation of this group of patients to ascertain the subsequent diagnostic yield.

Methods All patients were referred to a dedicated coeliac or gluten sensitivity clinic by GPs through a choose and book system. The referral criteria were "GI symptoms attributed to gluten ingestion." Investigations included baseline haematology, biochemistry, haematinics, $\mathrm{C}$ reactive protein and HLA status for the DQ2/DO8 haplotypes. In addition, coeliac serology was performed: endomysial antibody (EMA), tissue transglutaminase antibody (tTG), immunoglobulins, as well as duodenal biopsies on a gluten containing diet. A diagnosis of coeliac disease was based on either the presence of villous atrophy or in cases with lesser degrees of the modified Marsh grading, an associated positive coeliac serology and/or HLA typing.

Results 140 patients were investigated over a 5-year period. $80 \%$ were women and the median age of presentation was $37 \mathrm{yrs}$ (range 16-88). In patients self-reporting symptoms related to the ingestion of gluten, the diagnosis of coeliac disease was reached in $10 \%$ (n 14). 85\% (n 119) did not have coeliac disease but fulfilled the ROME criteria for irritable bowel syndrome (IBS). These patients were given a clinical diagnosis of gluten sensitive IBS (GS-IBS). Importantly, organic pathology was found in 5\% (n 7) all of whom had additional alarm symptoms-mesenteric ischaemia, bacterial overgrowth, lactose intolerance, bile salt malabsorption, lymphocytic colitis, ulcerative colitis and pyloric stricture. A positive coeliac serology $(p<0.0001$, exact fisher test) was significantly associated with coeliac disease. All patients with coeliac disease were HLA positive compared to $44 \%$ of GS-IBS cases. There was statistically no significant difference in gender, clinical symptoms or baseline bloods (haemoglobin, vitamin $\mathrm{B}_{12}$, folate, ferritin, calcium or albumin) between the groups, ( $p>0.05)$.

Conclusion Self-reporting of gluten related GI symptoms only results in a diagnosis of coeliac disease in $10 \%$ of cases. The majority of patients do not have overt coeliac disease but may belong to the spectrum of GS-IBS, a relatively new and under researched concept. Moreover, $5 \%$ had significant underlying disease. An empirical trial of gluten-free diet prior to referral may be unhelpful and delay the diagnosis in this group of patients. This data suggests that patients who self-report gluten induced GI symptoms should be considered for further investigations.

Competing interests None declared. 